\title{
A Survey on Big Five Personality Traits Prediction Using Tensorflow
}

\author{
Manisha Nilugonda $^{1}$, Dr. Karanam Madhavi ${ }^{2}$ \\ ${ }^{1}$ PG Scholar, Gokaraju Rangaraju Institute of Engineering and Technology, TS, India \\ ${ }^{2}$ Professor, Dept. of CSE, Gokaraju Rangaraju Institute of Engineering and Technology, TS, India
}

\begin{abstract}
A personality trait is a specific pattern of thought, thinking, or performing that manages to be faithful over time and beyond essential places. The Big Five-Extraversion, Agreeableness, Conscientiousness, Neuroticism and Openness to Practice are a set of five broad, bipolar quality dimensions that establish the most extensively used design of personality construction. Earlier investigations revealed a growing interest in defining the personality and behavior of people in fields such as career development, personalized health assistance, counseling, mental disorder analysis, and the detection of physical diseases with personality shift symptoms. Modern methods of discovering the Big-Five personality types include completing a survey, that takes an impractical amount of time and cannot be used often. This paper provides a survey on detecting of big five personality traits based on facial features recognition using TensorFlow mechanism. And also, various methods to detect big five personality traits are discussed in this paper. Finally, the graph provides a comparison between various detection of big five personality traits on facial expressions.
\end{abstract}

\section{INTRODUCTION}

Interpersonal communication skills and personality traits are diagnosed as critical compliance factors for overall activity performance and company effectiveness [Conrad et al. 2011 [1]]. Communication skills allow participants in the workplace to efficiently share rates and comment on records of different stakeholders through verbal and non-verbal messages [Spitzberg et al. 2007 [2]]. Verbal signals are used to convey specific phrases, facial expressions, non-verbal messages, which consist of gestures, and posture and tone of voice, are useful for obtaining information about underlying feelings, mentalities and feelings. Character developments correspond to the patterns of horror, attitudes, and behavior that can be used to wait if the character is excellent for a specific process context or organizational setting. Face-to-face interviews are an unusual way to choose jobs, and this approach is a valid assessment tool for measuring conversation skills between people in a prepared form. Interviewers may also decide to develop the candidate's personality based on the non-contact of the alternate at an unspecified time in the future during the interview, and the trial may have an additional impact on hiring proposals.
However, inviting all applicants to activities to attend nonpublic interviews is not cost-effective [Nikolaou et al. 2018 [3]]. Asynchronous Video Interview (AVI) has become an evolving alternative. Job applicants are required to log into an interview platform and submit their answers to predefined interview questions via webcam and microphone to your portable device or laptop. The solutions are analyzed using native citizens below. AVI allows candidates to submit and solve problems in any community and at any time.

Additionally, AVI speeds up selection because video interview statistics can be independently shared and evaluated for human evaluators' convenience without scheduling an interview. Some teachers or experts may be interested in knowing whether the interviews can be calculated to displace the traditional human evaluators entirely or in components if there are any criteria available to assess the overall performance of the interview in a known way. Popular strategies for advanced biometrics and face detection have been developed so that a pair of nearinfrared image patterns can be extracted with a tethered laptop. With the advent of artificial intelligence (AI), many laptop scientists are using preferred and accessible facial AI-based biometry technology to expand a fully computerized AVI-based digital interview platform (known as AVI-AI). AVI-AI technology has attracted considerable

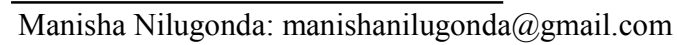


interest in the fields of computer science and human resources, primarily to assess communication skills and personal characteristics mechanically [Escalante et al. 2018 [4]]. Because AVI-AI is a one-use device of choice, to our knowledge, its authenticity and accuracy are still unknown. Artificial intelligence it is a computer technology division looking for a new type of smart device like human intelligence. Machine learning (ML) is a popular method of achieving artificial intelligence and in deep learning (DL) is a method used to put ML in practice. DL can routinely, instead of manually, perform feature extraction. There are three mainstream strategies for DL: supervised knowledge, uncensored learning, and semi-supervised knowledge. Research has shown that the semi-supervised field can be supplemented with tiny bits of facts without further tagging some of the categorized statistics for pattern recognition. Convolutional neural networks $(\mathrm{CNN})$ have been verified to be high-fashion costumes that can automatically classify patterns in image stats, and $\mathrm{CNN}$ is the most frequently used classifier that can be trained to precisely locate and capture facial impressions. Without extracting a feature manually, according to Sun et al. (2018) [5].

Previous Automatic personality recognition (APR) were introduced entirely based on supervised ML, which includes time-consuming marking guides. Since convolutional neural networks $(\mathrm{CNN})$ have proven to be a highly mobile innovation that can process shots mechanically and infer the first impressions from digital camera images, this examination conducted semi-supervised DL methods, consisting of $\mathrm{CNN}$, to increase artificial intelligence based on artificial intelligence and the corresponding agent who can understand The applicant is mechanically characteristic of a task using notably smaller datasets than candidates' facial expressions

Therefore, we use semi-DL and CNN-based classifieds based on TensorFlow to extend the AVI-AI that can routinely verify communication capabilities for job seekers and look forward to the immense 5-character features of the candidates as seen through actual interviews consistent with the facial features of the candidates. TensorFlow is a popular open source DL framework that can be grown in distinct heterogeneous structures across a variety of devices and platforms, including cell phones and laptops. As a result, the CNN TensorFlow framework is expected to fully reap the impact of massive facial recognition in the context of video interviews. This outlook tested the validity and accuracy of verbal talents of interpersonal exchange and took great directions in 5 people using AVI-AI.

\section{Comprehensive Analysis}

Recently, there have been many ways to reveal personality traits. One of them is APR. It mainly focuses on the Big Five Traits during interview process. This helps to identify characteristics and attributes from various signals without depending on self-assessment measures and also automatically assigns an individual's personality profile on their digital footprints.

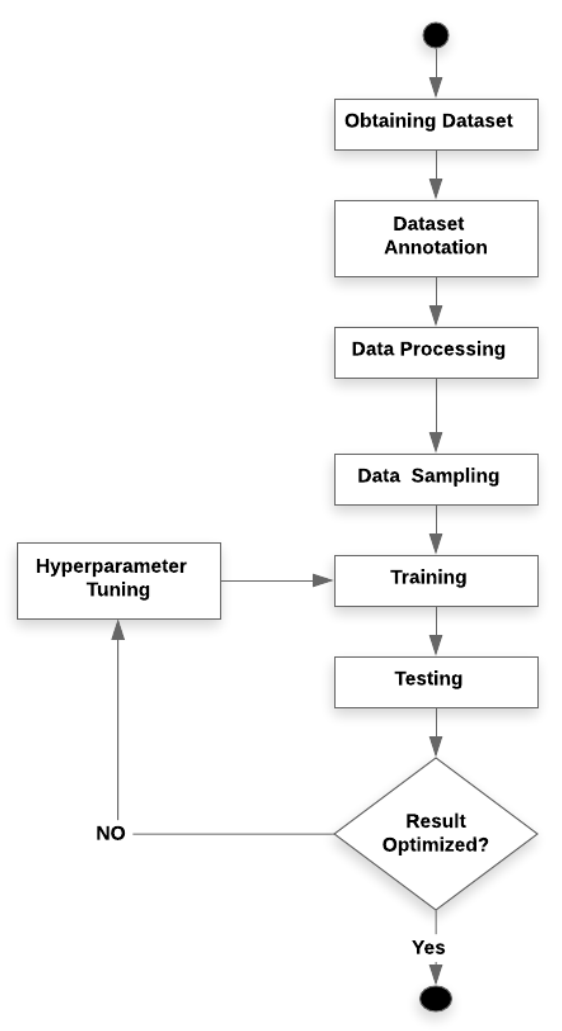

Fig.1 Block diagram of APR

By studying the voice of spoken conversations and relying mainly on aspects of the path and rhythm of speech, it is possible to record and understand personality trends or wait for the speaker's positions in a robotic manner. These tactics show that recordings are essential to the character. Below we discuss some popular personality styles.

McLarnon et al. (2019) [6] Personality measures are usually used in the selection of employees and various situations of excessive risk. In these settings, respondents can also interact with phishing or functional simulations to increase the likelihood of a valuable outcome (i.e., a realistic view). However, some humans also tend to fail a little, others may show more extreme response developments, and others may also respond. In this view, we use internal data from two waves of men or women to analyze counterfeiting as a measure of conscience, an honest reaction and copying conditions, and use latent transfer analysis (LTA) for specific types of counterfeiters. Kindness, anxiety, and perceived ability to deceive (PATD), acquired in the case of a sincere reaction, was used to assume irregular behavior patterns. We also tested whether counterproductive

Manisha Nilugonda: manishanilugonda $@$ gmail.com 
workplace behavior (CWB) differs between types of counterfeiting. The results supported three pending responses in each specific case and no response case, and responders could be described explicitly, moderately, false, and very flawed-accidental anxiety as predictors of energetic response patterns. PATD results do not now mean a broad predictive relationship to malicious behavior.

Asabere et al. (2018) [7] the search for self-confidence and socially conscious reference structures is being expanded to illustrate problems that do not show a recent start to blood. The relative importance of educational conferences led to the need for consulting systems seeking to establish guidelines for attending the conference. In this article, we intend to improve the accuracy of querying socially wise recommendation systems by providing a set of rules for written hybrid recommendations called personal and social counselors (PerSAR). PerSAR hybridizes the social and private behaviors of smart conference participants. Our approach to public consultations seeks to contract with an algorithm framework that calculates the similarities and unique strengths of those in the association. That way, sensitive and reliable leads can be created for them using a set of relevant facts. The experimental results verify that our proposed consulting approach is preferred and overrides various advanced and appropriate recommendation techniques and rules.

Celiktutan et al. (2017) [8] In this article, they have supported a new multimedia framework for automatically predicting impressions of extroversion, kindness, consciousness, nervousness, openness, attractiveness, and compassion regularly over time and in different situational contexts. Unlike the current work, we get more useful and practical visual annotations consistently overtime over the same set of topics, for the first time in literature, and evaluate them based on audible annotations. We recommend the use of a continuous prediction technique over time that learns temporal relationships rather than immediate treatment one after another. Our experiments show that quality prediction effects are received. At the same time, regression models are learned from audiovisual annotations and visual cues, and audiovisual annotations and visual cues mixed with acoustic signals in the degree of resolution. Always created annotations can provide a better view of the impressions that can be formed and anticipated more dynamically, and different from contextual context, which has seemed more consistent and robust over the years.

Brenner et al. (2016) [9] the predominant observation was to merge research findings of the attractiveness of the age with research on applicants' reactions to the new technology of the increasing process of selecting asynchronous video interviews. 106 volunteers conducted asynchronous video interviews and completed various questionnaires, as one of the candidates' personalities. In line with previous studies to learn about technology, the facts found that the perceived benefit and perceived ease of use predicted attitudes toward asynchronous video interviews. Moreover, openness revealed the modification of the relationship between the perceived benefit and attitudes toward this era of choice. No impressive results were found for computer self-efficacy, self-efficacy, job openness, openness, nervousness, and awareness. Theoretical and realistic effects are mentioned.

Jordan et al. (2015) [10] the machine learning was gained knowledge and addresses the issue of how to build computer systems that improve the technical area. It is one of the fastest-growing technical fields these days, located at the intersection of knowledge and technological information from computers, in artificial intelligence and data science. The recent development of machine learning has been driven by improved algorithms, machine learning theory, and the constant explosion of online information availability and occasional pricing calculations. The adoption of statistically intensive system mastery strategies can be discovered at some point in science, age and commerce, leading to a more fully evidence-based selection in many lifestyles, including health care, manufacturing, training, financial modeling, monitoring, advertising, and marketing.

Petrican et al. (2014) [11] the judgment of Characters, based on the appearance of the face, influence the personal choices and behaviors of the perceived person and goals. However, resistance to these consequences has not been determined for a more extended period of knowledge. To address this query, we had 51 differences between informers and informants for long-married spouses from the Big Five Inventory list. Participants were also filmed while being asked to maintain an emotionally neutral expression. A subset of the first pattern completed a short model of the Big Five stocks in response to shots of different heterosexual taxpayers (who were not familiar with them). The Oosterhof and Todorov's model of computer-based facial assessment was used to create facial feature categories for reliability, mastery, and style, which were mainly based on taxpayer photos. The results found that structural facial features are indicating increased reliability expected definitive biased international assessments of a target's personality, between both spouses and strangers. Between the spouses, this effect changed to water for the duration of the marriage.

Moreover, he became evidence indicating Dorian Gray's influence on the character, for the reason that the reliability of the face anticipated not the most straightforward pair and the stranger, but also the signs of particular flatness. The results of their gift recommend that widespread emotional cues, embedded in the form of emotionally neutral faces, lead to lasting results in personal judgments even among well-known goals and celebrities.

Nestler et al. (2013) [12] this article has been imposed the latest research on the utility and expansion of a lens copy to 
interpersonal judgment. They first explained how to use the basic lens version to describe the methods underlying the accuracy of known own experiences. Then, described how that model could be distinguished by its understanding of studies on intuitive and reflexive judgments, implicit and explicit personality controls, and behavior managed in one form or another. Also, proceeded to combine research into lens version with judgment errors and experience updates. Finally, the authors described extensions of the lens copy to various personality phenomena to 0 known, along with the Meta accuracy and taste. In general, this review indicates that lens issuance is a convincing and flexible framework that can be used to understand personal judgments.

Batrinca et al. (2012) [13] the person's personality in the human-computer interaction (HCI) played a critical position in the general compliance of the conversation. The modern look has become a significant advantage by automatically revealing the developments of the Big Five character, from the minimum movies of two to five minutes, because the computer interacts with precise levels of cooperation to stimulate the arrival of those personal trends. Emotional stability and coup d'etat were the two basic principles for adequately organizing different private cooperative organizations. All configurations of emotional stability and medium, non-cooperative arrangements were at all flat. Interestingly, friendship and knowledge could be smoother under a rather useless web page. Finally, our homework does not seem to spark many creative behaviors.

Batrinca et al. (2011) [14] Personality has played a significant role in the way humans control the images they had conveyed in private shows and job interviews, seeking to influence first impressions of the alternative and increased effectiveness. This document was examined the automatic detection of the evolution of the big five traits from small electronic screens (30 one hundred and twenty seconds), by investigating the effectiveness of 29 nonverbal visual and auditory properties. Our effects show that awareness / emotional/neurotic stability are fun and recognized traits. The lower levels of accuracy of flatness and friendliness are explained by the interaction between detailed features and the differential activation of underlying behavioral trends in traits.

Htike et al. (2010) [15] the popularity of the human condition was gained more and more attention in the areas of artificial intelligence and computer fantasy due to its promising packages in the areas of personal health care, environmental recognition, human-computer interaction, and surveillance systems. Getting to know the human condition in video footage is a difficult challenge and is part of the single biggest problem with interpreting video footage. In this article, a smart system was proposed to recognize the human position in video sequences. First, the system has become an educated and valuable categorize of five unique social sites in using every acquired knowledge that was supervised and not controlled by works. The supervised classifier became using a multilayer perceptron (MLP), Feed forward Neural Networks at the same time as the Unsupervised Classifiers, and Self-organizing Maps (SOM), Fuzzy C-Means (FCM) and K-Mean clustering. The results indicate that MLP plays (96\% accuracy) significantly higher than SOMs, FCM and K Means, which provide $86 \%, 33 \%$ and $31 \%$ accuracy, respectively. Second, all classifiers have been classified and reassessed to classify jobs. With the two best locations, the accuracy of all works is greatly expanded, especially for uncensored works. This indicated that advanced cognitive classifier subject to supervision progress to non-censored for the reputation of the task of human position and that censored workbooks no longer examine cases in which many jobs are learned compared to the supervised knowledge workbook with high accuracy in any case.

DeGroot et al. (2009) [16] The purpose of this test examines the role of a person's traits in understanding relationships between nonverbal cues and performance evaluations of known interviews. Design / Technology The behavior-based interview has changed to a higher level to identify the manipulation of a significant employer across the country. Managers were interviewed, and video interviews recorded $(\mathrm{n}=$ one hundred and ten). These films have been used as catalysts for citizens in this view. Results indicate that evaluators will obtain personality traits using the most straightforward statistical channel, and these traits provide in part, an explanation of the relationships between nonverbal cues and performance metrics.

Moreover, the awareness features explain the relationship between visual cues and dozens of interviews, and the flatness features mediate the relationship between audio signals and dozens of meetings. Neurotic characteristics had a severe effect on both visual and acoustic signals. Implications Regardless of how well the dependency is for the interview, non-verbal references are intended for the discussions to make attribution for applicants. If we encounter this fact, instead of remembering signal records as a bias to be overlooked, interviews can do a better process of specializing in behavior and job-related data within the conversation, where they discover that the scores provide statistics that have to attend. Originality / Value This study took the record resources provided to assessors to the audio or video channel to study their impact from my point of view. A version of the Brunswick lens indicates the ability of the character traits to expect both activity and interview performance in general when using the two data channels.

Zheng et al. (2008) [17] Previous research recommended generalizing the cultural movement from a five-element structure to personality traits. In this text, we analyze the program for two variations (one hundred objects and 50 elements) of IPIP Big-Five factor markers in both

Manisha Nilugonda: manishanilugonda $@ g m a i l . c o m$ 
heterosexual samples $(\mathrm{N}=633)$ and homosexuals $(\mathrm{N}=437)$ samples in China. The evaluation of factors within the differences confirmed that each version of the IPIP measurements showed clean, orthogonal structures of 5 components almost identical to the American shape in each of the problem samples. The reliability of the five elements was very high, except for the 50-element gauge. The entire component correlations between 100 and 50 features were excessive, as were the component match parameters between heterosexual and same-sex samples. Both variants of IPIP Big-Five aspect indicators were closely related to the Big Five Inventory Metrics (BFI: John, Donahue \& Kentle, 1991), thus conveying some simultaneous validation in the Chinese context.

\section{Various Methodologies to Determine Big Five Personality Traits}

The characteristics of the Big Five are a strategy to determine the effect of behavioral functions in humans with this release. The results demonstrated a vital component in predicting human behavior based on this model and also on the hobby of characters.

\subsection{Facial Action Coding system (FACS)}

To determine the type of character, Big-Five has many questions that seek to take an irrelevant time and are now not used consistently repeatedly. In this file, this stage focuses on reading a new, unused approach that expects BFPT through facial expressions that received from FACS. At the forefront of this, the relationship between the movement apparatus that includes the extremes of facial expressions is shown by the particular individual's behavior. Moreover, this approach provides a good result for estimating introductory behavior, extroversion and nervousness show real results in a short time, while in contrast to the time used in the questionnaire technique to evaluate facial expressions, FACS is one of the scientific findings of methods that usually evaluate unspeakable intentions through subtle expressions [Gavrilescu 2015].

\subsection{Positive and Negative Affect Schedule (PANAS)}

The evaluation of the mutual information (MI) general performance between various methods described like, (i) excitation (ii) equivalence (iii) personality dimensions, and PANAS. The analysis indicates that implicit responses are beneficial to the emotional and personal nation/individuals of PANAS. Therefore, personality / PANAS attributes can be detected in the presence of feelings. The main results can be summarized as follows:
(I) The three methods involve excessive reciprocal verbs with the size of the person and PANAS, but the relationship is usually not linear.

(2) The peripheral physiological functions have much better reciprocal events with the range of the Big Five trends and PANAS than other suggested features

(3) Due to the linear strength of the direct family members between (a) EEG and openness and (b) peripheral and diastolic physiological warnings, we ended up with implicit high scores in $\mathrm{F} 1$ (about $70 \%$ ) in predicting a diastole / high/low with simple linear technique [Abadi et al 2015].

\subsection{Fuzzy buried Markov model (FBMM)}

In the following investigation, each emotion occurs through a specific muscle within the face. A specific UA becomes almost certain information about the emotional world, roughly, for a specific element. Each unmarried face movement involves spreading AUs as an additive or nonadditive form. For every single business unit that defines a multi-state facial model, the face is divided into five elements: eyes, cheeks, eyebrows, lips, and wrinkles. In the study phase, the model with some frameworks covering facial procedures is obtained in impressive statistical responses. A few movies are built to predict the six feelings (joy, disgust, happiness, anger, fear, and terror) [Zhan et al.2010].

\subsection{Fully Automatic Facial Action Unit Detection and Temporal Analysis (FAUDT)}

Each video has a much shorter duration. Therefore, the copy was oriented within the real arrangement of a safe and varied range of tires. Ultimately put everything towards the number one element familiar with the face through the base set, which is VJFD. The observed face is divided into five components through this algorithm. Each component divided into this educational body receives similar classes from that class. It means whether AU (Action unit) is a gift or not, as well as the effectiveness of the extension. The compilations mentioned above are already expert and given maximum precision. From the previous description, the presence of AU from I to $\mathrm{V}$ was rated to build a standardized property that provides a behavior map. Every component of the behavior map that led to the modification relies mainly on the outcomes of the nervous community, to reduce the error rate in the following diffusion method. Neural network results are measured by a result obtained from the model, and it is a questionnaire. In this testing phase, real-time records are obtained from this typical subject. Each purchased object was passed to a VJFD rule set, and then the divided element was moved into blocks, showing whether AU was present or not after that, and checking the effect.

Societal neural behavior maps across confined rows to restrict treatment time. Each row is derived from this process from the steps that await the individual behaviors during these stages. Being open to experience: an

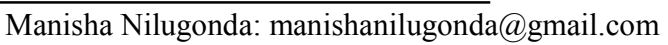


adventurous, emotional, and extraordinary ideology, appreciation for the network will become intriguing. Consciousness: Depends on others, self-discipline; For achievement, a willfulness of willful behavior that avoids spontaneous behavior will be prepared below. Flatness: reassurance, caused by the employer to others, nice feelings, the conversation will fall under this category. Satisfactory: Mercy, which is no longer suspicious, helps nature and temperate will come under this chapter. Neuroticism: Anxiety and unpleasant feelings will be easily enjoyed, and a lack of instinct to control comes under this category [Valstar et al.2006].

\subsection{Support Vector Machine (SVM)}

Support Vector Machine (SVM) is a supervised learning algorithm analyzes the information and detection styles used for the type. SVM takes the educational records set and marks it as part of a category, then predicts whether or not the verification report is a member of modern magnificence. SVM fashions constitute records as a point in space divided with the help of a line/ hyperplane. The hyperplane's optimal search characteristic is shown in the problem to Scoring in SVM to find the use of the verification document class.

Table.1 Comparison between various methods to determine big-five personality traits

\begin{tabular}{|c|c|c|c|c|}
\hline $\begin{array}{l}\text { Authors with } \\
\text { year }\end{array}$ & Methodology & Achievement & Pros & Cons \\
\hline $\begin{array}{l}\text { [Gavrilescu et } \\
\text { al.2015 [18]] }\end{array}$ & $\begin{array}{lr}\text { Facial } & \text { Action } \\
\text { Coding } & \text { System } \\
(\text { FACS }) & \end{array}$ & $\begin{array}{l}\text { This system proved its } \\
\text { strength in recognizing } \\
\text { extroversion and nervousness } \\
\text { and presented the } \\
\text { consequences of the accuracy } \\
\text { of openness to enjoying } \\
\text { personality traits. }\end{array}$ & $\begin{array}{l}\text { FACS movement } \\
\text { tools provide the } \\
\text { maximum intensity of } \\
\text { facial functions and } \\
\text { personal inclinations } \\
\text { for men or women, } \\
\text { and the machine } \\
\text { performs over } 90 \% \\
\text { accuracy }\end{array}$ & $\begin{array}{l}\text { He became unable, as he } \\
\text { should be, to decide } \\
\text { conscientiously and } \\
\text { pleasantly. }\end{array}$ \\
\hline $\begin{array}{l}\text { [Abadi et al } \\
2015[19]]\end{array}$ & $\begin{array}{lr}\text { Positive } & \text { and } \\
\text { Negative } & \text { Affect } \\
\text { Schedule } & \end{array}$ & $\begin{array}{l}\text { Studied the relationship } \\
\text { between the personal, } \\
\text { primary, and personal } \\
\text { emotional agenda and the } \\
\text { implicit responses of humans } \\
\text { to sensitive content materials. }\end{array}$ & $\begin{array}{l}\text { All the hired } \\
\text { modalities proportion } \\
\text { high data with the } \\
\text { persona and PANAS } \\
\text { dimensions. }\end{array}$ & $\begin{array}{l}\text { Incomplete answers and } \\
\text { biases in the answers are } \\
\text { for their reasons. }\end{array}$ \\
\hline $\begin{array}{l}\text { [Zhan et } \\
\text { al.2010 [20]] }\end{array}$ & $\begin{array}{ll}\text { Fuzzy } & \text { buried } \\
\text { Markov } & \text { model } \\
(\text { FBM) } & \end{array}$ & $\begin{array}{l}\text { Solved classical recognition } \\
\text { model problem }\end{array}$ & $\begin{array}{l}\text { FBMM has a faster } \\
\text { training } \\
\text { speed, affinity } \\
\text { popularity rate, and } \\
\text { more migher } \\
\text { strength in popular } \\
\text { facial expressions } \\
\text { below image streams. }\end{array}$ & $\begin{array}{l}\text { It rarely satisfied in } \\
\text { facial expression } \\
\text { recognition }\end{array}$ \\
\hline $\begin{array}{l}\text { [Valstar et } \\
\text { al.2006 [21]] }\end{array}$ & \begin{tabular}{lr} 
Fully & Automatic \\
Facial & Action \\
Unit Detection \\
and & Temporal \\
\multicolumn{2}{c}{ Analysis }
\end{tabular} & $\begin{array}{l}\text { A combination of scouting } \\
\text { strategies based on the } \\
\text { evaluation of vertical and } \\
\text { horizontal graphs of the upper } \\
\text { and lower half of the facial } \\
\text { region imaging accomplishes } \\
\text { this. }\end{array}$ & $\begin{array}{l}\text { An advantage of } \\
\text { feature selection by } \\
\text { Gentle Boost is that } \\
\text { features are selected } \\
\text { contingent on the } \\
\text { features that have } \\
\text { already been selected }\end{array}$ & $\begin{array}{l}\text { At this point, it is best to } \\
\text { move and adjust the } \\
\text { facial features' positions } \\
\text { in the semi-front view } \\
\text { images. } \\
\text { There is no independent } \\
\text { guidance for detecting } \\
\text { and controlling the face's } \\
\text { facial factors. }\end{array}$ \\
\hline $\begin{array}{l}\text { [Lin Li et } \\
\text { al.2014 [22]] }\end{array}$ & $\begin{array}{l}\text { Support Vector } \\
\text { Machine (SVM) }\end{array}$ & $\begin{array}{l}\text { It analyzed the information } \\
\text { and detection styles used for } \\
\text { the type }\end{array}$ & $\begin{array}{l}\text { SVM is part of a } \\
\text { category and then } \\
\text { predicts whether the } \\
\text { verification report is a } \\
\text { member of the most } \\
\text { advanced group. }\end{array}$ & $\begin{array}{l}\text { Wasting all the } \\
\text { important time that is } \\
\text { limited in most cases }\end{array}$ \\
\hline
\end{tabular}

Manisha Nilugonda: manishanilugonda $@ g m a i l . c o m$ 


\section{COMPARISON RESULTS}

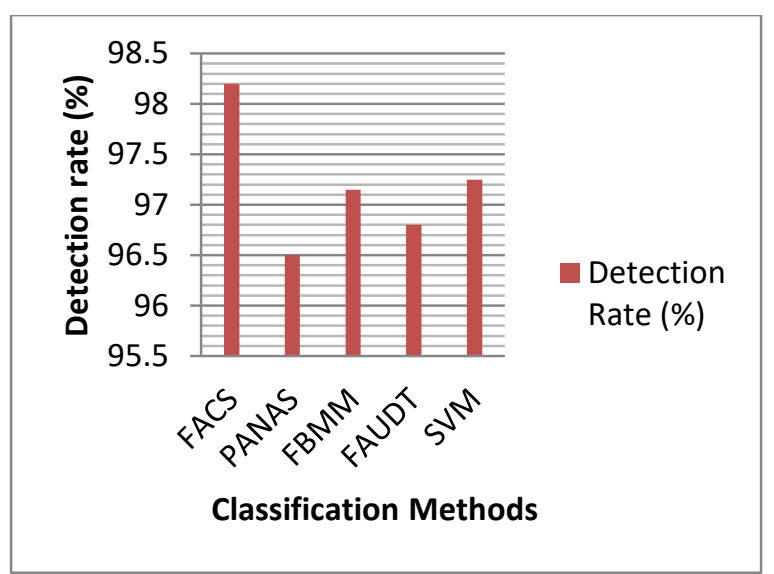

Fig.2 Detection rate Vs Classifiers

As shown in figure 2, the various methods to recognition big five personality traits are compared with the current classifiers of FACS, PANAS, FBMM, FAUDT, and Support vector machine (SVM)

\section{CONCLUSION}

Personality traits are usually distinguished by intense monitoring, which protects many factors such as pregnancy and ability. There are many instructions for influencing the people categorized under these behaviors. Building a new system can determine the difficulty of character trends entirely based on facial functions. This non-surgical device may be more sensitive to use in professional development, health aids, and diagnosis of mental health problems or physical illnesses with symptoms of personality change. This paper provided a brief survey on detecting big five personality traits on facial expression. Also, it presented various methods to identify the big five personality traits. Finally, a comparison between different ways for the detection of big five personality traits is given.

\section{REFERENCES}

1. D Conrad, R Newberry, 2011, “24 business communication skills: attitudes of human resource managers versus business educators." pp.:4-23.

2. BH Spitzberg, Adams, 2007, "CSRS, the conversational skills rating scale: an instructional assessment of interpersonal competence".

3. Nikolaou I, K Foti, 2018, "Personnel selection and personality", pp 659-677.

4. Escalante HJ, Junior JJ, 2018, "Explaining first impressions: modeling, recognizing, and explaining apparent personality from videos". arXiv preprint arXiv :18020 0745.

5. Sun A, Li Y, Lu G, 2018, "Facial expression recognition using optimized active regions", HumCentric Comput Inf Sci 8:33.

6. McLarnon M. J. W and T. J. Schneider, 2019, "Faking it! Individual differences in types and degrees of faking behavior,"pp. 88-95.

7. Asabere N. Y and M. B. Michael, 2018, "Improving socially-aware recommendation accuracy through personality," pp. 351-361.

8. O. Celiktutan and H. Gunes, 2017, "Automatic prediction of impressions in time and across varying context: personality, attractiveness and likeability," pp. 29-42.

9. F. S. Brenner, T. M. Ortner, and D. Fay, 2016, "Asynchronous video interviewing as a new technology in personnel selection: the applicant's point of view," pp. 1-11.

10. M. I. Jordan and T. M. Mitchell, 2015, "Machine learning: trends, perspectives, and prospects," pp. 255260.

11. R. Petrican, A. Todorov, and C. Grady, "Personality at face value: facial appearance predicts self and other personality judgments among strangers and spouses," J. Nonverbal Behavior, vol. 38, no. 2, pp. 259-277, Jan. 2014.

12. S. Nestler and M. D. Back, 2013, "Applications and extensions of the lens model to understand interpersonal judgments at zero acquaintance,", pp. 374-379.

13. L. Batrinca and F. Pianesi, 2012,"Multimodal recognition of personality traits in human-computer collaborative tasks," pp. 39-46.

14. L. M. Batrinca, N. Sebe, 2011,"Please, tell me about yourself: automatic personality assessment using short self-presentations,", pp. 255-262.

15. K. K. Htike and O. O. Khalifa, 2010, "Comparison of supervised and unsupervised learning classifiers for human posture recognition,", pp. 1-6

16. Gavrilescu, M, 2015, "Study on determining the BigFive personality traits of an individual based on facial expressions", pp. 1-6.

17. Abadi, I. Sebe, N, 2015, "Inference of personality traits and affect schedule by analysis of spontaneous reactions to affective videos", pp. 1-8.

18. Yogeswara Reddy B, Srinivas Rao J, Suresh Kumar T, Nagarjuna A, International Journal of Innovative Technology and Exploring Engineering, 8(11), 2019, pp: 1194-1198.

19. Valstar, M., Pantic, M, 2006, "Fully automatic facial action unit detection and temporal analysis", p. 149.

Manisha Nilugonda: manishanilugonda $@$ gmail.com 
20. T. DeGroot and J. Gooty, 2009, "Can nonverbal cues be used to make meaningful personality attributions in employment interviews?", pp. 179-192.

21. Suresh Kumar T, Sankar V, Reliability improvement of composite electric power system using Unified Power Flow Controller 2011 IEEE India Conference, INDICON, 2011.

22. Lin Li, 2014, "Predicting Active Users' Personality Based on Micro-Blogging Behaviors", pp.1-11

23. L. R. Goldberg, "The development of markers for the big-five factor structure," Psychological Assessment, vol. 4, no. 1, pp. 26-42, Mar. 1992.

24. Ramesh, G., Madhavi, K, Surekha. P, "Feature Selection for Effective Heart Disease Prediction Models towards Data Science ", Communicated to Ingénierie des Systèmes d'Information Journal April 2020.

25. Thirupathi, N., Madhavi, K., Ramesh, G., Sowmya Priya, K. Data Storage in Cloud Using Key-Policy Attribute-Based Temporary Keyword Search Scheme (KP-ABTKS), Lecture Notes in Networks and Systems, 2020.

26. Ramesh, G., Madhavi, K., Best keyword set recommendations for building service-based systems
International Journal of Scientific and Technology Research, October, 2019.

27. Ramesh, G., Madhavi, K. Summarizing Product Reviews using NLP based Text Summarization", International Journal of Scientific \& Technology Research, September 2019.

28. Ramesh, G., Madhavi, K. An Efficient and Effective Framework to Track, Monitor, and Orchestrate Resource Usage in an Infrastructure as a Service, International Journal of Recent Technology and Engineering (IJRTE) ISSN: 2277-3878, Volume-8 Issue-3, September 2019. DOI:10.35940/ijrte.C5690.098319.

29. Ch. Mallikarjuna Rao, G. Ramesh, Madhavi, K., "Feature Selection Based Supervised Learning Method for Network Intrusion Detection", International Journal of Recent Technology and Engineering (IJRTE), ISSN: 2277-3878, Volume-8, Issue-1, May 2019.

30. Madhavi, K., G. Ramesh, G. Lavanya, "Load effectiveness on coverage-technique for test case prioritization in regression testing", International Journal of Innovative Technology and Exploring Engineering (IJITEE) ISSN: 2278-3075, Volume-8 Issue-7 May, 2019. 